\title{
Aberrant expression of the glycolytic enzymes aldolase B and type II hexokinase in hepatocellular carcinoma are predictive markers for advanced stage, early recurrence and poor prognosis
}

\author{
SHIAN-YANG PENG ${ }^{1}$, PO-LIN LAI ${ }^{2}$, HUNG-WEI PAN ${ }^{3}$, \\ LI-PIN HSIAO ${ }^{2}$ and HEY-CHI HSU ${ }^{2,3}$ \\ ${ }^{1}$ Department of General Education, National Taipei College of Nursing; ${ }^{2}$ Department of \\ Pathology, National Taiwan University Hospital; ${ }^{3}$ Graduate Institute of Pathology, \\ College of Medicine, National Taiwan University, Taipei, Taiwan, R.O.C.
}

Received October 22, 2007; Accepted December 3, 2007

\begin{abstract}
Cancer cells with a high glycolytic rate have an advantage in tumor growth. Hepatocellular carcinoma (HCC) often exhibits an aberrant expression of glycolytic enzymes, particularly type II hexokinase (HKII) and aldolase B (ALDOB). This study examined the aberrant expression of HKII and ALDOB in 203 surgically resected HCCs. A dramatic down-regulation of ALDOB was found in 116 HCCs $(57 \%)$, while $43 \%$ of HCCs maintained the expression. HKII mRNA was overexpressed in $70(35 \%)$ primary HCCs. The ALDOB down-regulation and HKII overexpression correlated with high-grade (grade II-IV) HCC (all $\mathrm{ps}<0.0001)$, portal vein invasion (stage IIIB-IV) $\left(\mathrm{ps}<1 \times 10^{-6}\right)$, early tumor recurrence $($ ETR $)(p<0.001$ and $p<0.01$, respectively) and a lower 5-year survival ( $\mathrm{p}=0.000001$ and $\mathrm{p}=0.0062$, respectively). Notably, in stage II HCC which had no vascular invasion, the ALDOB down-regulation was associated with ETR $(\mathrm{p}<0.05)$ and a lower 5-year survival $(\mathrm{p}=0.015)$. The down-regulation of ALDOB correlated with a high AFP $\left(\mathrm{p}=1 \times 10^{-8}\right)$, whereas the overexpression of HKII, which has two functional motifs for the mutant p53, correlated with the p53 mutation, $\mathrm{p}<0.01$. The three factors (ALDOB down-regulation, HKII overexpression and p53 mutation) not
\end{abstract}

Correspondence to: Dr Shian-Yang Peng, Department of General Education, National Taipei College of Nursing, Taipei 100, Taiwan, R.O.C.

E-mail: peng@ntcn.edu.tw

Dr Hey-Chi Hsu, Department of Pathology, National Taiwan University Hospital, 7 Chung-Shan South Road, Taipei 100, Taiwan, R.O.C.

E-mail: heychi@ntu.edu.tw

Key words: hepatocellular carcinoma, aldolase B, type II hexokinase, portal vein invasion, early tumor recurrence, prognosis only correlated with tumor progression, but also interacted with one another, leading to a more aggressive HCC with a portal vein invasion and various extent of intrahepatic metastasis by more than four-fold $\left(\mathrm{ps}<1 \times 10^{-6}\right)$ and frequent ETR by more than two-fold (ps<0.0001) compared with HCCs without the events. In conclusion, the aberrant expression of ALDOB and HKII is associated with advanced disease, ETR and poor prognosis, and ALDOB down-regulation in stage II HCC is a predictive marker of ETR and an unfavorable outcome.

\section{Introduction}

During the progression of malignant tumors, the genetic and epigenetic alterations accumulate and the rapidly growing tumors need to overcome hypoxia and nutrient deprivation owing to the inadequate blood supply, leading to changes in the energy demands of the cancer cells $(1,2)$. The metabolic changes are manifested by an increase in glucose, fatty acid and amino acid metabolism and a decrease in oxidative phosphorylation $(3,4)$. Glucose is the primary energy source and a high rate of glycolysis, which is one of the earliest discovered hallmarks of cancer cells $(3,5,6)$, provides the tumor with metabolic and survival advantages. Several key genetic alterations associated with tumor development were recently shown to affect glycolysis directly, such as p53 mutation and the activation of HIF-1 $(7,8)$. The two bona fide tumor suppressor mitochondrial enzymes, succinate dehydrogenase (SDH) and fumarate hydratase (9), are parts of the Krebs cycle that links glucose metabolism to mitochondria oxidative phosphorylation. The accumulation of succinate caused by SDH mutation leads to the stabilization and activation of HIF-1 $\alpha$ (8). The tumor suppressor p53 activates TIGAR (TP53-induced glycolysis and apoptosis regulator) to block glycolysis. Thus, the inactivation of p53 would decrease respiration and increase glycolysis (10). The unique high glycolytic metabolic phenotype in cancer has become a new potential target for gene therapy $(11,12)$, tumor imaging and the prediction of therapeutic effects (13-15). These observations highlight the importance of the glycolytic enzymes in human cancer. 
Hepatocellular carcinoma (HCC), the leading cause of cancer mortality in Taiwan (16), has unique features distinct from other human cancers. The liver is the biggest metabolic organ in the body with enriched metabolic enzymes, and receives mainly hypoxic portal venous blood enriched with nutrients from the gastrointestinal absorption, whereas the highly vascular HCC receives a predominantly arterial blood supply. The dramatic changes in blood and oxygen supply, together with the shut-off of the privileged nutrients from the portal vein blood, will inevitably force HCC to develop alternatives, particularly the glycolytic enzymes, to adapt these changes (17). The crucial role of glucose utilization for HCC cell growth is highlighted by the observation that HCC cell lines undergo acute cell death upon glucose starvation $(18,19)$ and the replacement of glucose by pyruvate represses glycolytic enzyme activity and inhibits rat hepatoma cell proliferation (17).

The glycolytic enzymes, hexokinase and aldolases, perform the first and sixth step of glycolysis, respectively. Many cancers with a high glucose catabolic rate frequently depend on the overexpression of type II hexokinase (HKII), especially under hypoxic conditions (20). HKII, which is nearly silent in the liver due to DNA hypermethylation $(21,22)$, is markedly overexpressed in highly glycolytic tumors $(21,23-25)$, including the human HCC (26) and HCC cell lines $(21,24,27)$. HKII has two functional p53 motifs in the promoter, which can be activated by the mutant p53, and plays a role in maintaining a high glycolytic rate in p53-mutated cancer cells (28). Aldolase catalyzes the reversible cleavage of fructose-1,6-(bis) phosphate and fructose 1-phosphate to dihydroxyacetone phosphate and either glyceraldehyde-3phosphate or glyceraldehyde, respectively $(29,30)$. Vertebrate aldolases have three isozymes. Aldolase A (ALDOA) exists in muscle and fetal liver, aldolase B (ALDOB) predominantly in adult liver, and aldolase $\mathrm{C}$ in the brain. The fetal liver isozyme aldolase A increased after a partial hepatectomy and in hepatoma, whereas the more differentiated type isozyme aldolase $\mathrm{B}$ did not change and was distinctly low in hepatoma $(30,31)$.

Regardless of these observations, the general consensus is mainly based on the observations in cancer cell lines $(21,23,24,32)$, and due to the information in human HCC being limited to a small series of patients the clinicopathological significance is largely unknown (33). In this study, we analyzed the aberrant expression of the two major glycolytic enzymes, ALDOB and HKII, in 203 HCCs to elucidate their clinicopathological significance. We showed that the aberrant expression correlated with vascular invasion, early tumor recurrence (ETR) and the poor prognosis of HCC, and interacted with each other and with the p53 mutation, towards advanced disease.

\section{Materials and methods}

Patients. Between 1982 and 1997, 203 surgically resected, unifocal, primary HCCs, which were pathologically assessed at the National Taiwan University Hospital and had RNA samples, formed the basis of this study, as described (34-36). These patients had an adequate liver function reserve and had not received intervention therapies, as previously described
(34-36). This study was executed according to the regulation of the ethics committee of the Hospital. The patients were 156 males and 47 females, with a mean age of 54.9 (range, 14-88) years. Serum hepatitis B surface antigen (HBsAg) was detected in 134 cases, anti-HCV antibody in 62, including 15 positive for both. Liver cirrhosis was found in 81 cases (40\%).

Histological study and tumor staging. The tumor grade was divided into three groups: well-differentiated (grade I, 49 cases), moderately-differentiated (grade II, 88 cases) and poorly-differentiated (grade III, 59 cases and grade IV, 20 cases). The tumor stage of the unifocal HCC closely correlated with prognosis, as described $(35,36)$. Stage IIIA-IV HCC had vascular invasion, whereas stage I and II HCC did not. The tumor staging was classified into I (well-encapsulated minute HCC $\leq 2 \mathrm{~cm}, 4$ cases), II (encapsulated or unencapsulated HCC without vascular invasion, 84 cases) and IIIA (with invasion of vessels in tumor capsule, 32 cases). Stage IIIB (25 cases) had an invasion of a portal vein (PV) branch, accompanied by a grossly discernible or microscopic satellite close to the tumor in 19 cases $(76 \%)$. Stage IV HCCs (58 cases) had an invasion of the major PV branches and were accompanied by satellites deep in the liver in 51 cases (88\%), or an invasion of the Gleason capsule or the tumor rupture (1 case).

Reverse transcription-polymerase chain reaction ( $R T-P C R)$. RT-PCR assays at the exponential phases were used for large-scale quantitative measurements for human ALDOB, HKII and the internal control S26 ribosomal protein mRNA in an automated DNA thermal cycler 480 (Perkin-Elmer/ Cetus), as described (35-38). The PCR was stopped at 22 cycles for ALDOB and S26, and at 30 for HKII with an initial additional 8 cycles for HKII. The target mRNA expression levels on $2 \%$ agarose gel were determined by the ratios of signal intensity of the target genes to that of S26 measured by 1D Image Analysis Software (Kodak Digital Science, USA). The target (ALDOB or HKII)/S26 ratios were scored as high $(>1.2,49$ cases and 34 cases respectively), intermediate (0.5-1.2, 38 and 36 cases, respectively), low $(<0.4,13$ and 81 cases, respectively) and negligible or negative (103 and 52 cases, respectively). A ratio of $<0.5$ at 22 PCR cycles was considered as the down-regulation for ALDOB, and a ratio of $\geq 0.5$ was regarded as the overexpression for HKII.

Primers for PCR reactions. The PCR reaction primers are: AldoB-F: 5'-ATG CCA CTC TCA ACC TCA ATG CTA TC3'; AldoB-R: 5'-TTA TTT TCT TGG GTG GGT ATT CTG G-3'; Hex2-F1701: 5'-GAG GCG CCT TCT AGC AGT TG-3'; Hex2-R2184: 5'-TCC AAG ATC CAG AGC CAG GA-3'; S26-F: 5'-CCG TGC CTC CAA GAT GAC AAA G-3' and S26-R: 5'-GTT CGG TCC TTG CGG GCT TCA C-3'.

Tissue culture and conditions. All the cell lines, including HCC cell lines (Hep3B, HA22T, PLC/PRF/5, SKHEp-1 and HepG2) were cultured in DMEM supplement with $10 \%$ fetal bovine serum at $37^{\circ} \mathrm{C}$ with $5 \% \mathrm{CO}_{2}$.

Analysis of p53 mutation. The mutations of the p53 tumor suppressor gene were analyzed in 169 tumors by a direct 


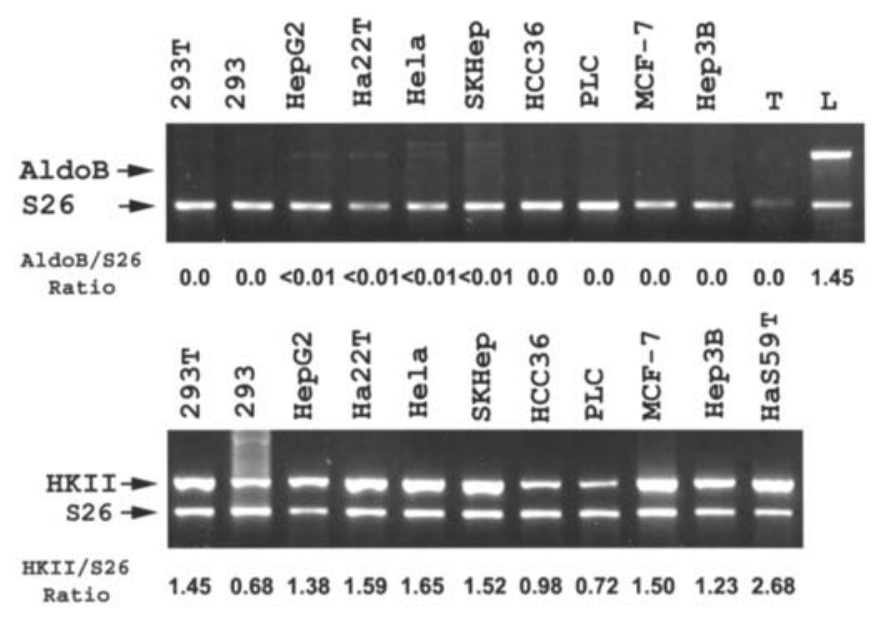

Figure 1. Expression of Aldolase B (ALDOB) and type II hexokinase (HKII) in cell lines (upper panel). With RT-PCR at the linear range, ALDOB mRNA was undetectable or expressed at very low levels in all the hepatocellular carcinoma (HCC) (HA22T, SKHep, HCC36, PLC/PLZ/5 and Hep3B) and hepatoblastoma (HepG2) cell lines, and the cell lines of non-liver cell lineage (MCF-7, HeLa, HEK293 and HEK293T). T and L, paired human HCC and liver RNA samples (lower panel). HKII mRNA was expressed in all the cell lines examined.

sequencing spanning exon 2 to -11 , as previously described $(39,40)$

Follow-up observation, early tumor recurrence (ETR) and treatment. The intrahepatic tumor recurrence or distant metastasis detected by imaging diagnosis within 12 months after tumor resection was designated as early tumor recurrence (ETR), as previously described $(35,36)$. Among the 203 study patients, 202 cases $(99.5 \%)$ were followed for $>5$ years $(71$ cases) or until death (131 cases), and 184 (90\%) were eligible for the evaluation of ETR. Among the 89 cases with ETR, $40(45 \%)$ received tumor resection and/or TACE.
Statistical analysis. The data analyses were carried out using the Epi Info (Version 3.3.2, Centers for Disease Control and Prevention, Atlanta, GA) and SAS (Version 9.1, SAS Institute Inc., Cary, NC) software. The $\chi^{2}$, Fisher exact test and log-rank test were used for univariate analysis.

\section{Results}

Expression of $A L D O B$ and HKII in human hepatocellular carcinoma (HCC) and HCC cell lines. The mRNA expression levels of ALDOB and HKII in human HCC cell lines (Fig. 1) and paired $\mathrm{HCC}$ and non-tumorous liver tissue samples (Figs. 2 and 3) were examined by RT-PCR at the linear range, 22 cycles for ALDOB, 30-32 for HKII and 22 for internal control ribosomal protein mRNA S26. The ALDOB mRNA was expressed at a high level in the human liver (Fig. 2), but was undetectable or expressed at very low levels in all the cancer cell lines of liver cell lineage (HA22T, HCC36, Hep3B, PLC/PRF/5, SKHEp and HepG2) (Fig. 1) and frequently decreased dramatically in human HCC (Fig. 2). In contrast, HKII mRNA was detected in all the cancer and immortalized cell lines of liver and non-liver cell lineage (Fig. 1, lower panel). In human HCC, HKII mRNA was often overexpressed, but mostly at lower amounts compared with the cell lines.

Among the 203 unifocal, primary HCCs examined, ALDOB mRNA was dramatically decreased or absent in 116 (57\%) of the samples (Fig. 2), while the down-regulation was found in only $9(5 \%)$ out of 181 the non-tumorous livers examined. The overexpression of HKII was detected in 70 (35\%) of the 203 HCCs (Fig. 3). However, HKII mRNA was also overexpressed in $19(13 \%)$ out of the 145 non-tumorous livers examined.

Aberrant expression of $A L D O B$ and HKII in HCC: clinicopathological significance. Among the clinical features, HCCs with ALDOB down-regulation were strongly associated with high AFP level (>200 ng) as compared with those without

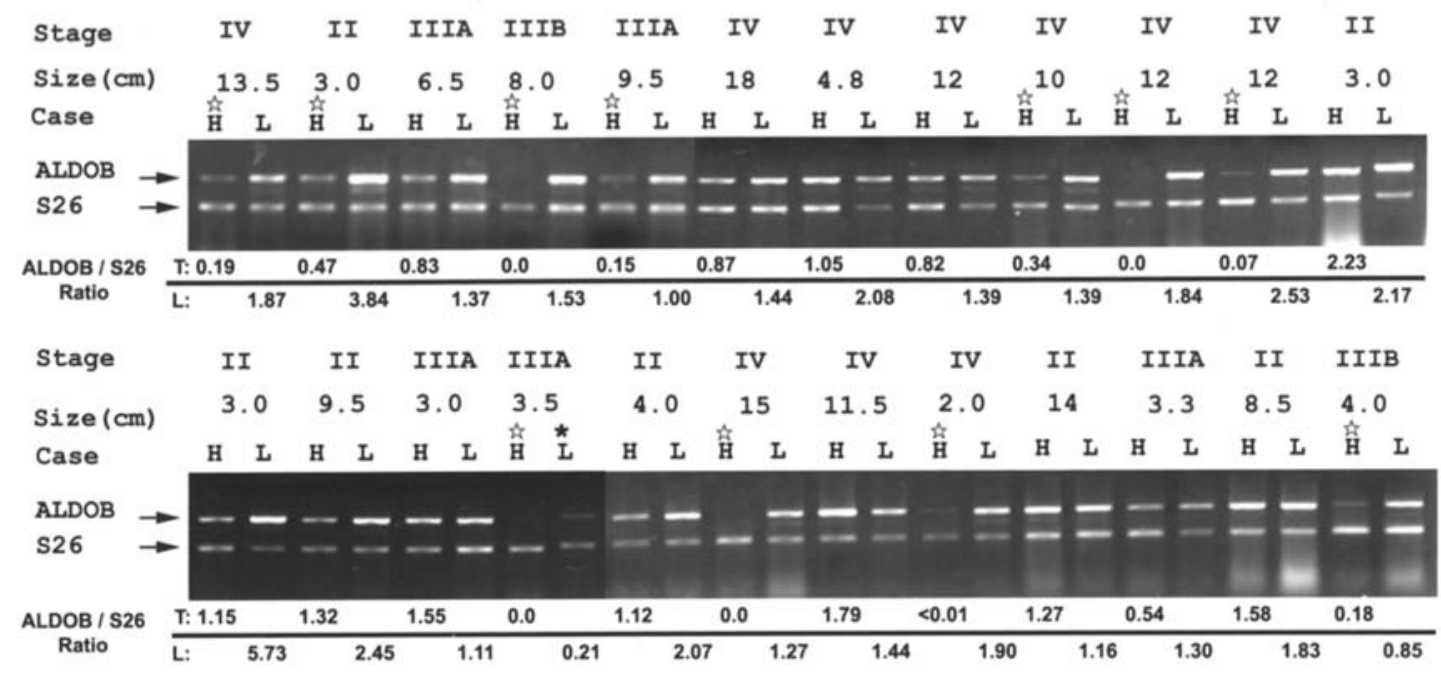

Figure 2. Expression of ALDOB in human HCC measured by RT-PCR. The mRNA levels of ALDOB were significantly decreased (ALDOB/S26<0.5) in 11 $($ 간) out of $24 \mathrm{HCC}(\mathrm{T})$ and in one paired non-tumorous liver $(*)$. S26, ribosomal protein mRNA as internal loading control. Tumor stage and tumor size are listed above and the ALDOB/S26 ratios for tumor and liver below. 
Table I. The down-regulation of ALDOB in relation to major clinicopathological indices of tumor progression in unifocal hepatocellular carcinomas.

\begin{tabular}{|c|c|c|}
\hline & $\mathrm{ALDOB} \downarrow^{\mathrm{a}}$ & HKII $\uparrow$ \\
\hline Total & $\mathrm{N}(\%)$ OR $(95 \% \mathrm{CI})$ & $\mathrm{N}(\%)$ OR $(95 \% \mathrm{CI})$ \\
\hline
\end{tabular}

\begin{tabular}{|c|c|c|c|c|c|}
\hline \multicolumn{6}{|c|}{ Mean age (years) } \\
\hline$>55$ & 108 & $56(52)$ & 1.0 & $28(26)$ & 1.0 \\
\hline$\leq 55$ & 95 & $60(63)$ & $1.6(87-2.9)$ & $42(44)$ & $2.26(1.2-4.3)^{\mathrm{d}}$ \\
\hline \multicolumn{6}{|l|}{ Sex } \\
\hline Male & 156 & $85(55)$ & 1.0 & $52(34)$ & 1.0 \\
\hline Female & 47 & $31(66)$ & $1.6(0.78-3.38)$ & $17(36)$ & $1.09(0.54-2.36)$ \\
\hline \multicolumn{6}{|c|}{ Serum HBsAg } \\
\hline Negative & 69 & $33(48)$ & 1.0 & $16(23)$ & 1.0 \\
\hline Positive & 134 & $83(62)$ & $1.78(0.95-3.3)$ & $24(31)$ & $2.24(1.1-4.5)^{\mathrm{c}}$ \\
\hline \multicolumn{6}{|c|}{ AFP (ng/dl) } \\
\hline$\leq 200$ & 105 & $40(38)$ & 1.0 & $30(29)$ & 1.0 \\
\hline$>200$ & 98 & $76(78)$ & $5.6(2.9-10.9)^{\mathrm{h}}$ & $40(41)$ & $1.7(0.92-3.2)$ \\
\hline \multicolumn{6}{|c|}{ Liver cirrhosis } \\
\hline Negative & 122 & $65(53)$ & 1.0 & $44(36)$ & 1.0 \\
\hline Positive & 81 & $51(63)$ & $1.49(0.8-2.8)$ & $26(32)$ & $0.84(0.44-1.6)$ \\
\hline \multicolumn{6}{|c|}{ Tumor size $(\mathrm{cm})$} \\
\hline$\leq 5$ & 87 & $45(52)$ & 1.0 & $31(32)$ & 1.0 \\
\hline$>5$ & 116 & $71(61)$ & $1.12(0.6-2.1)$ & $44(38)$ & $1.43(0.76-2.7)$ \\
\hline \multicolumn{6}{|c|}{ Tumor grade: } \\
\hline $\mathrm{I}$ & 39 & $11(28)$ & 1.0 & $3(8)$ & 1.0 \\
\hline II-IV & 164 & $105(64)$ & $4.53(1.99-10.5)^{\mathrm{f}}$ & $67(41)$ & $8.3(2.3-35.3)^{\mathrm{f}}$ \\
\hline \multicolumn{6}{|c|}{ Tumor stage } \\
\hline I-II & 88 & $32(36)$ & $1.0^{\mathrm{i}}$ & $16(18)$ & $1.0^{\mathrm{j}}$ \\
\hline IIIA & 32 & $21(66)$ & $3.26(1.3-8.4)^{\mathrm{i}}$ & $8(25)$ & $1.5(0.52-4.5)^{\mathrm{j}}$ \\
\hline IIIB-IV & 83 & $63(76)$ & $5.51(2.7-11.4)^{\mathrm{i}}$ & $46(55)$ & $3.23(1.46-7.2)^{\mathrm{j}}$ \\
\hline \multicolumn{6}{|l|}{$\mathrm{ETR}^{\mathrm{b}}$} \\
\hline No & 95 & $40(42)$ & 1.0 & $23(24)$ & 1.0 \\
\hline Yes & 89 & $61(69)$ & $3.0(1.6-5.8)^{\mathrm{e}}$ & $41(46)$ & $2.67(1.36-5.3)^{\mathrm{d}}$ \\
\hline \multicolumn{6}{|c|}{ p53 mutation } \\
\hline No & 84 & $41(49)$ & 1.0 & $21(25)$ & 1.0 \\
\hline Yes & 85 & $50(59)$ & $1.5(0.78-2.88)$ & $38(45)$ & $2.43(1.2-4.9)^{\mathrm{d}}$ \\
\hline
\end{tabular}

aParenthesis indicates percentage. $\downarrow$ and $\uparrow$, designate down-regulation and overexpression, respectively. ${ }^{b}$ Early tumor recurrence designates intrahepatic tumor recurrence or distant metastasis detected within 12 months after tumor resection. P-value: ${ }^{\mathrm{c}}<0.05$; ${ }^{\mathrm{d}}<0.01 ;{ }^{\mathrm{e}}<0.001$; ${ }^{\mathrm{f}}<0.0001 ;{ }^{\mathrm{g}}<0.000001 ;{ }^{\mathrm{h}} 1 \times 10-8$; ${ }^{\mathrm{i}}$ stages IIIA-IV versus stages I-II, $\mathrm{p}<0.000001 ;{ }^{\mathrm{j}}$ stages IIIB-IV versus stages I-IIIA, $\mathrm{p}<0.000001$.

the down-regulation ( $\leq 200 \mathrm{ng}$ ), but not without other parameters, such as age, gender and serum HBsAg status (Table I). Pathologically, the down-regulation of ALDOB positively correlated with a higher tumor grade (grade II-IV HCC, $\mathrm{p}<0.00001$ ), but not with liver cirrhosis and notably tumor size. Importantly, the down-regulation of ALDOB was strongly associated with a more advanced stage (IIIB-IV) of HCC $\left(\mathrm{p}<1 \times 10^{-8}\right)$, as compared with stage I-II HCC (Table I).
The overexpression of HKII mRNA, which correlated with a younger age $(\mathrm{p}<0.01)$ and positive serum HBsAg $(\mathrm{p}<0.05)$, did not correlate with serum AFP elevation. Histopathologically, HKII overexpression also correlated with highgrade (grade II-IV) tumors $(\mathrm{p}<0.0001)$ and, importantly, was also associated with a higher tumor stage (IIIB and IV), which was more than three-fold higher than stage I and II tumors $\left(\mathrm{p}<1 \times 10^{-8}\right)$. 


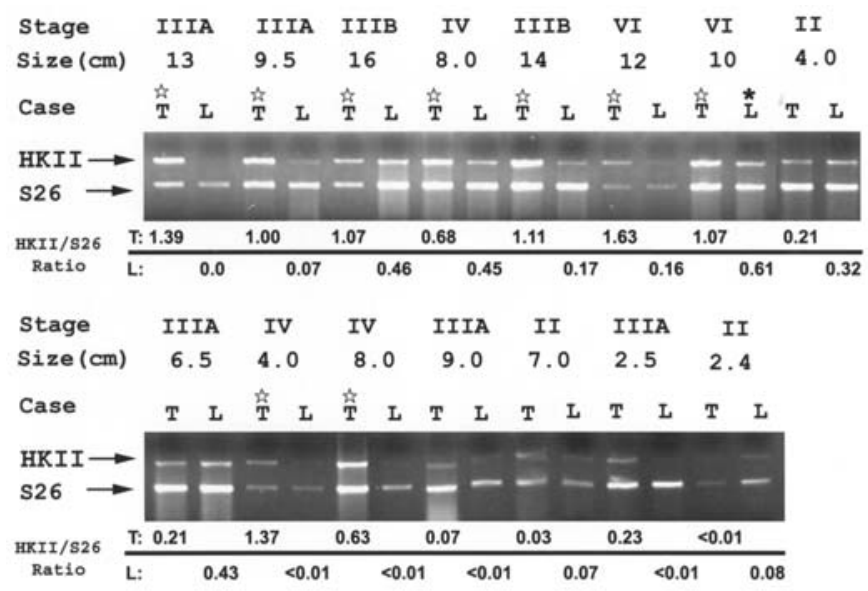

Figure 3. Expression of HKII in human HCC measured by RT-PCR. The mRNA levels of HKII were overexpressed (HKII/S26>0.5) in 9 (ㄱ) out of 15 HCCs $(\mathrm{T})$ and in one liver $(*)$. Note that HKII was often expressed in the non-tumorous livers, although at lower levels. S26, ribosomal protein mRNA as an internal loading control. Tumor stage and tumor size are listed above and the HKII/S26 ratios for tumor and liver below.

$A L D O B$ down-regulation and HKII overexpression in relation to early tumor recurrence (ETR) and poor prognosis. ETR is the most crucial clinical event associated with the poor prognosis of HCC patients after receiving total tumor resection $(35,41,42)$ and is considered a critical unfavorable prognostic event ahead of death. Thus, the aberrant expression of ALDOB and HKII was compared with other clinicopathological risk factors for ETR. As shown in Table II, ALDOB down-regulation ( $\mathrm{p}=0.00042)$, HKII overexpression $(\mathrm{p}=0.0016)$, high AFP $(\mathrm{p}=0.000024)$ and $\mathrm{p} 53$ mutation $(\mathrm{p}=0.0005)$ were significant molecular risk factors. Besides, the stage IIIA tumor $(\mathrm{p}=0.00051)$, stage IIIB-IV tumor $\left(\mathrm{p}=1 \times 10^{-8}\right)$ and larger tumor $(\mathrm{p}=0.00031)$ were the significant histopathological risk factors, particularly stage IIIB-IV tumors that had invasion of portal vein (PV) branches and were accompanied by a grossly discernible or microscopic satellite tumor nodule in $87 \%$ cases ( 72 out of 83 ).

Importantly, the down-regulation of ALDOB and overexpression of HKII, which were associated with highgrade and high-stage HCCs and ETR, contributed to lower rate of 5 -year survival, $\mathrm{p}=0.000001$ and $\mathrm{p}=0.0062$, respectively (Fig. 4A and $\mathrm{B}$ ).

The down-regulation of $A L D O B$ in low-stage HCC predicts ETR and poor prognosis. Although the tumor stage is the most crucial histopathological prognostic factor of HCC (35), the patients of the same tumor stage often have a different prognosis. To determine whether the aberrant expression of HKII and ALDOB can predict the patients of the same tumor stage but with a higher risk of poor prognosis, we analyzed the gene expression in the HCC of different stages. In stage II HCC, the down-regulation of ALDOB was associated with more frequent ETR $(32 \%$ or $9 / 28$ versus $13 \%$ or $7 / 52, \mathrm{p}<0.05)$ and a lower rate 5-year survival than those with a normal ALDOB expression, $\mathrm{p}=0.015$ (Fig. 4C).

Interaction of the aberrant expression of ALDOB and HKII with p53 mutation in relation to portal vein invasion and
A

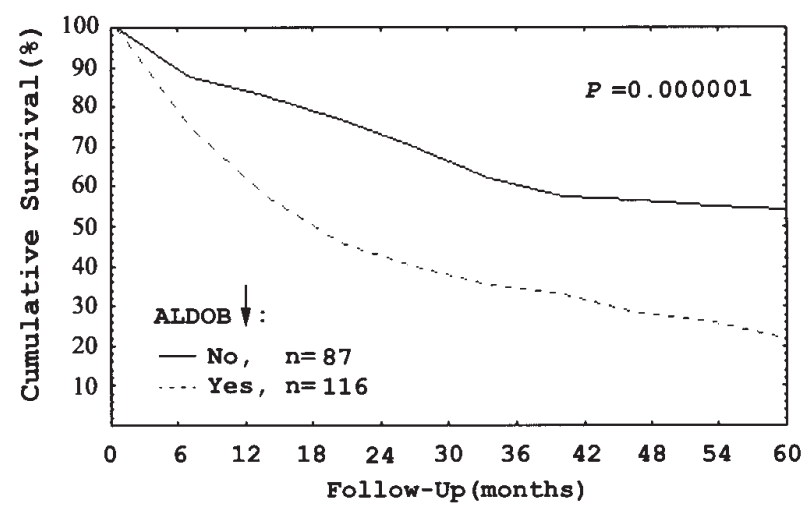

B

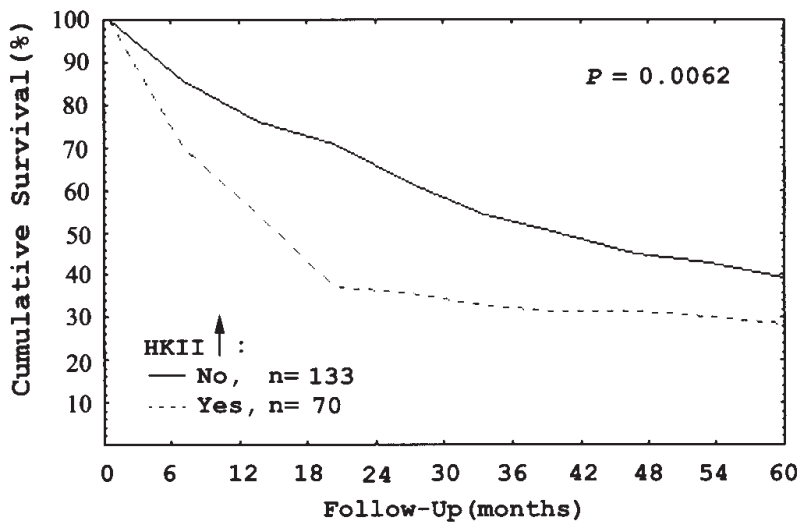

C

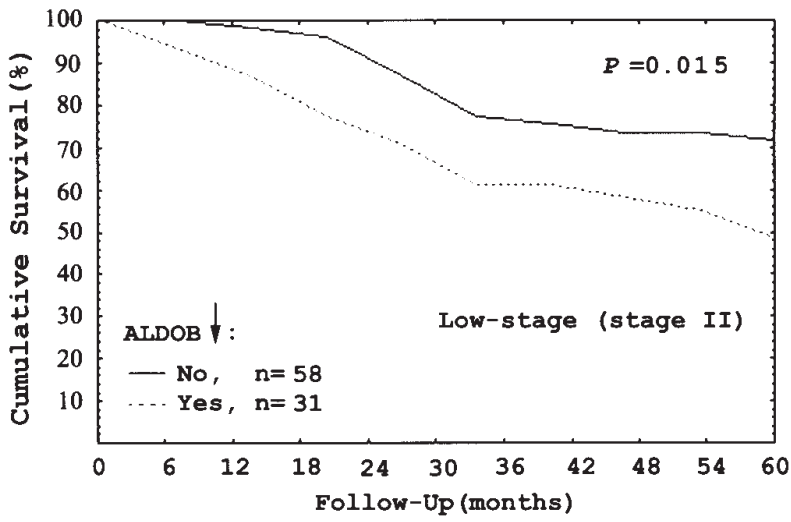

Figure 4. Expression of aldolase B (ALDOB) and type II hexokinase (HKII) in hepatocellular carcinoma in relation to cumulative survival. $\mathrm{HCC}$ with the down-regulation of ALDOB (A) and overexpression of HKII (B) had a lower 5 -year survival, $p=0.000001$ and $p=0.0062$, respectively. In low-stage (stage II) HCC (C), the down-regulation of ALDOB was associated with a lower rate 5-year survival, $\mathrm{p}=0.015$ (log-rank test).

ETR. The promoter of HKII contains two functional p53 motifs and is activated by the mutant p53 (28). In this study we found that p53 mutation, which was detected in 85 (50\%) out of 169 HCCs examined, correlated with HKII overexpression $(\mathrm{p}<0.01)$, but not with ALDOB down-regulation (Table I). We therefore carried out pair-wise analysis to explore the potential interaction of the aberrant expression of ALDOB and HKII with p53 mutation. The aberrant expressions of the two genes exhibited an interaction with each other and with the p53 mutation and contributed cooperatively toward more 
Table II. Risk factors for early tumor recurrence.

\begin{tabular}{|c|c|c|c|c|}
\hline & \multirow[b]{2}{*}{ Total } & \multicolumn{2}{|c|}{ Early tumor recurrence } & \multirow[b]{2}{*}{ p-value } \\
\hline & & $\mathrm{N}(\%)$ & OR $(95 \%$ CI $)$ & \\
\hline \multicolumn{5}{|c|}{ Age (years) } \\
\hline$>55$ & 94 & $39(41)$ & 1.0 & \\
\hline$\leq 55$ & 91 & $50(55)$ & $1.7(0.92-3.21)$ & 0.067 \\
\hline \multicolumn{5}{|l|}{ Sex } \\
\hline Male & 140 & $64(46)$ & 1.0 & \\
\hline Female & 45 & $25(56)$ & $1.48(0.72-3.08)$ & 0.25 \\
\hline \multicolumn{5}{|l|}{ HBsAg } \\
\hline Negative & 65 & $25(38)$ & 1.0 & \\
\hline Positive & 120 & $64(53)$ & $1.83(0.95-3.55)$ & 0.053 \\
\hline \multicolumn{5}{|l|}{ Cirrhosis } \\
\hline Yes & 71 & $32(45)$ & 1.0 & \\
\hline No & 114 & $57(50)$ & $1.22(0.64-2.31)$ & 0.514 \\
\hline \multicolumn{5}{|c|}{ Tumor size (cm) } \\
\hline$\leq 5$ & 79 & $24(30)$ & 1.0 & \\
\hline$>5$ & 106 & $65(61)$ & $3.63(1.87-7.09)$ & 0.00031 \\
\hline \multicolumn{5}{|c|}{ Tumor grade } \\
\hline I & 38 & $7(18)$ & 1.0 & \\
\hline II-IV & 134 & $74(55)$ & $5.46(2.12-14.7)$ & 0.00006 \\
\hline \multicolumn{5}{|c|}{ Tumor stage ${ }^{a}$} \\
\hline I & 4 & $0 \quad(0)$ & - & \\
\hline II & 80 & $16(20)$ & 1.0 & \\
\hline IIIB-IV & 101 & $73(72)$ & $10.4(4.9-22.4)$ & $1 \times 10^{-8}$ \\
\hline \multicolumn{5}{|c|}{ AFP (ng/ml) } \\
\hline$\leq 200$ & 96 & $31(32)$ & 1.0 & \\
\hline$>200$ & 89 & $58(65)$ & $3.58(1.88-6.84)$ & 0.000024 \\
\hline \multicolumn{5}{|c|}{ p53 mutation } \\
\hline No & 77 & $24(31)$ & 1.0 & \\
\hline Yes & 78 & $46(59)$ & $3.17(1.56-6.5)$ & 0.0005 \\
\hline \multicolumn{5}{|c|}{ ALDOB down-regulation } \\
\hline No & 83 & $28(34)$ & 1.0 & \\
\hline Yes & 102 & $61(60)$ & $2.92(1.52-5.6)$ & 0.00042 \\
\hline \multicolumn{5}{|c|}{ HKII overexpression } \\
\hline No & 121 & $48(40)$ & 1.0 & \\
\hline Yes & 64 & $41(64)$ & $2.71(1.38-5.34)$ & 0.0016 \\
\hline
\end{tabular}

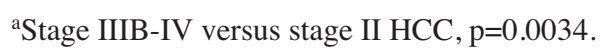

frequent stage IIIB and IV tumors and ETR. As shown in Table III, portal vein invasion (stage IIIB and IV) and ETR were low in HCC without the aberrant expression of ALDOB and HKII, but increased by more than four- and two-fold, respectively, in $\mathrm{HCC}$, with aberrations of the two genes ( $\mathrm{p}<1 \times 10^{-7}$ and $\mathrm{p}<0.0001$, respectively). Almost identical results were observed for the p53 mutation and ALDOB down-regulation as well as p53 mutation and HKII overexpression (Table III).

\section{Discussion}

HCC arises from the largest, highly vascular and relatively hypoxic metabolic organ, the liver and has some unique features distinct from other human cancers. HCC receives predominantly arterial blood from the hepatic artery but is shut off from the hypoxic although nutrients enrich portal blood for the liver, which inevitably forces the cancer cells to make a metabolic switch to ensure the energy sources. In this study 
Table III. Interaction between the aberrant expression of ALDOB and HKII and p53 mutation in relation to vascular invasion.

\begin{tabular}{|c|c|c|c|c|c|c|}
\hline & \multicolumn{3}{|c|}{ Stage IIIB-IV } & \multicolumn{3}{|c|}{ ETR } \\
\hline & Total & $\mathrm{N}(\%)$ & OR $(95 \% \mathrm{CI})$ & Total & $\mathrm{N}(\%)$ & OR $(95 \% \mathrm{CI})$ \\
\hline \multicolumn{7}{|c|}{ HKII $\uparrow /$ ALDOB $\downarrow$} \\
\hline $\mathrm{No} / \mathrm{No}$ & 69 & $11(16)$ & 1.0 & 66 & $20(30)$ & 1.0 \\
\hline $\mathrm{No} / \mathrm{Yes}$ & 64 & $26(41)$ & $3.6(1.49-8.86)^{\mathrm{b}}$ & 55 & $28(51)$ & $2.41 .06-5.4)^{\mathrm{a}}$ \\
\hline Yes/No & 18 & $9(50)$ & $5.3(1.5-19)^{\mathrm{b}}$ & 17 & $8(47)$ & $2.0(0.61-6.9)$ \\
\hline Yes/Yes & 52 & $37(71)$ & $13.0(5.0-35.0)^{\mathrm{e}}$ & 47 & $33(70)$ & $5.4(2.23-13.4)^{\mathrm{d}}$ \\
\hline \multicolumn{7}{|c|}{ p53 mutation / ALDOB $\downarrow$} \\
\hline No/No & 43 & $5(12)$ & 1.0 & 42 & $9(21)$ & 1.0 \\
\hline $\mathrm{No} / \mathrm{Yes}$ & 41 & $17(41)$ & $5.4(1.6-19.4)^{\mathrm{b}}$ & 35 & $15(43)$ & $2.8(0.91-8.4)^{\mathrm{a}}$ \\
\hline Yes/No & 35 & $13(37)$ & $4.5(1.3-16.9)^{\mathrm{b}}$ & 32 & $16(50)$ & $3.7(1.2-11.5)^{\mathrm{b}}$ \\
\hline Yes/Yes & 50 & $40(80)$ & $30.4(8.5-118)^{\mathrm{e}}$ & 46 & $30(65)$ & $6.9(2.4-20.2)^{\mathrm{d}}$ \\
\hline \multicolumn{7}{|c|}{ p53 mutation / HKII $\uparrow$} \\
\hline No/No & 63 & $11(17)$ & 1.0 & 58 & $17(29)$ & 1.0 \\
\hline $\mathrm{No} / \mathrm{Yes}$ & 21 & $11(52)$ & $5.2(1.6-17.6)^{\mathrm{b}}$ & 19 & $7(37)$ & $1.4(0.41-4.75)$ \\
\hline Yes/No & 47 & $15(32)$ & $2.2(0.83-6.08)$ & 43 & $21(49)$ & $2.3(0.94-5.7)^{\mathrm{a}}$ \\
\hline Yes/Yes & 38 & $27(71)$ & $11.6(4.1-34.3)^{\mathrm{e}}$ & 35 & $25(71)$ & $6.0(2.2-17.1)^{\mathrm{d}}$ \\
\hline
\end{tabular}

p-value: ${ }^{\mathrm{a}}<0.05 ;{ }^{\mathrm{b}}<0.01 ;{ }^{\mathrm{c}}<0.001 ;{ }^{\mathrm{d}}<0.0001 ;{ }^{\mathrm{e}} 1 \times 10^{-7}$.

of the expression of the two important glycolytic enzymes ALDOB and HKII in 203 surgically resected unifocal primary HCCs, ALDOB mRNA expression was significantly decreased in $116(57 \%)$ and maintained in the remaining cases $(43 \%)$, whereas HKII mRNA was overexpressed in $70(34 \%)$ primary HCCs. These alterations are in agreement with the suggestion that HCC has to use alternative metabolic pathways for energy sources crucial for tumor growth because of the dramatic microenvironmental changes. Although the frequent aberrant expression of ALDOB and HKII has been reported in human HCC $(26,33)$, the clinical or pathological significance is not clear. We showed that the down-regulation of ALDOB was the predominant change of the glycolytic enzymes in the human HCC because of its relative abundance in the adult liver and the frequent and dramatic decrease in HCC. Theoretically, the down-regulation of ALDOB would hamper glucose utilization and suppress tumor growth, and thus tumor metastasis. However, we showed that ALDOB downregulation was associated with high grade (II-IV, $\mathrm{p}<0.0001$ ) stages and, importantly, a more aggressive stage IIIB-IV HCC $(\mathrm{p}<0.000001)$. These findings suggest that HCC has successfully developed alternative paths for energy sources, such as the overexpression of HKII and ALDOA. The ALDOA overexpression was found in $45 \%$ of 203 HCCs and often in the HCC-bearing livers, but the overexpression did not correlate with tumor progression (data not shown).

HKII serves as one of the strategies for cancer cells to maintain efficient glucose utilization $(23,27)$ to assure a growth advantage under hypoxic conditions in rapidly growing tumors (20). HKII was expressed in almost all the cancer and immortalized cells, the HKII overexpression was less frequent $(35 \%)$ and the mRNA expression levels lower in human HCC compared with HCC cell lines. Besides, the non-tumorous livers, which had chronic hepatitis and/or liver cirrhosis, also had various degrees of HKII expression, with evident overexpression in $13 \%$. Regardless of its relatively low frequency, HCC with HKII overexpression had a higher grade II-IV $(\mathrm{p}<0.000001)$ and stage IIIB-IV tumor $(\mathrm{p}<0.000001)$ than HCC without the overexpression. Since stage IIIB-IV HCCs had a portal vein invasion and tumor satellites indicative of intrahepatic metastasis in $87 \%$ of the cases, these results were consistent with the suggestion that HKII expression contributes to HCC progression $(20,43)$. Taken together, our findings suggest that ALDOB downregulation and HKII overexpression are important biomarkers associated with more advanced HCC and not simply epiphenomena of tumor growth.

Despite improvements in patient management, the longterm prognosis of HCC patients after surgical resection remains grave, mainly because of the early tumor recurrence (ETR). ETR is the most crucial clinical factor predictive of poor prognosis and $<20 \%$ of the HCC patients with ETR can survive for $>5$ years $(35,36)$. Hence, molecular markers useful for the identification of high risk patients are needed. In this study, we showed for the first time that $\mathrm{HCC}$ with ALDOB down-regulation or HKII overexpression had an increased risk for ETR, $\mathrm{p}<0.0001$ and $\mathrm{p}<0.01$, respectively, and served as new molecular risk factors for ETR, in addition to a high AFP $(\mathrm{p}<0.0001)$ and p53 mutation $(\mathrm{p}<0.001)$. Consistent with the association with advanced tumor stage and frequent ETR, HCC with ALDOB down-regulation or HKII overexpression had a significantly lower 5-year survival rate, $\mathrm{p}=0.000001$ and $\mathrm{p}=0.0062$, respectively. Although a high tumor stage is the most important histopathological risk 
factor for the poor prognosis of HCC (35), the patients of the same tumor stage often have a different prognosis, particularly in patients of the low-stage tumor that had no vascular invasion and thus fewer histological markers for the prediction of prognosis. We found that ALDOB downregulation identified a subset of stage II HCC patients with a higher risk for ETR $(\mathrm{p}<0.05)$ and a poor prognosis $(\mathrm{p}=0.015)$, but not the overexpression of HKII.

The mutation of the p53 gene is one of the most common genetic aberrations in tumorigenesis (44-46). The p53 mutation can promote tumor cell growth and exhibit an oncogenic gain of function (47-50). Importantly, the p53 mutation is associated with a more aggressive HCC $(39,40)$. The promoter of HKII contains two functional p53 motifs and is activated by mutant p53 (28). We showed that HKII overexpression positively correlated with p53 mutation $(\mathrm{p}<0.01)$, but the ALDOB down-regulation did not. This finding may explain in part the relatively lower frequency of HKII overexpression in human HCCs, in which $<50 \%$ harbor a p53 mutation, compared with HCC cell lines which mostly have a p53 mutation. Due to their association with HCC progression, we then performed a pairwise analysis to explore the interaction between these three genetic and epigenetic alterations (ALDOB down-regulation, HKII overexpression and p53 mutation). We showed that they interacted with one another. Furthermore, HCC with any combination of two events had more stage IIIB-IV tumors by more than four-fold (all ps $<1 \times 10^{-7}$ ) and ETR by more than two-fold (all ps<0.0001), as compared with HCCs with neither of the two events.

In summary, we provided in vivo evidence linking the aberrant expression of ALDOB and HKII to an enhanced metastatic potential of HCC, ETR and the poor prognosis of HCC. The down-regulation of ALDOB helped to identify subsets of low-stage HCC patients with a poor prognosis.

\section{Acknowledgements}

This work was supported by the grants from the National Science Council and National Health Research Institute of the Republic of China, Taiwan (NSC95-2320-B-227-001 to SYP; NSC95-3112-B-002-007 to H-C Hsu). We thank Hauman Technologies Corp., Taipei, Taiwan for the partial financial support.

\section{References}

1. Wang GL, Jiang BH, Rue EA and Semenza GL: Hypoxia-inducible factor 1 is a basic-helix-loop-helix-PAS heterodimer regulated by cellular O2 tension. Proc Natl Acad Sci USA 92: 5510-5514, 1995.

2. Guillemin $\mathrm{K}$ and Krasnow MA: The hypoxic response: huffing and HIFing. Cell 89: 9-12, 1997.

3. Warburg O: On respiratory impairment in cancer cells. Science 124: 269-270, 1956 .

4. Haussinger D and Schliess F: Glutamine metabolism and signaling in the liver. Front Biosci 12: 371-391, 2007.

5. Beckner ME, Stracke ML, Liotta LA and Schiffmann E: Glycolysis as primary energy source in tumor cell chemotaxis. J Natl Cancer Inst 82: 1836-1840, 1990.

6. Greiner EF, Guppy M and Brand K: Glucose is essential for proliferation and the glycolytic enzyme induction that provokes a transition to glycolytic energy production. J Biol Chem 269: 31484-31490, 1994.

7. Dang CV and Semenza GL: Oncogenic alterations of metabolism. Trends Biochem Sci 24: 68-72, 1999.
8. Selak MA, Armour SM, MacKenzie ED, et al: Succinate links TCA cycle dysfunction to oncogenesis by inhibiting HIF-alpha prolyl hydroxylase. Cancer Cell 7: 77-85, 2005.

9. Gottlieb E and Tomlinson IP: Mitochondrial tumour suppressors: a genetic and biochemical update. Nat Rev 5: 857-866, 2005.

10. Green DR and Chipuk JE: p53 and metabolism: Inside the TIGAR. Cell 126: 30-32, 2006.

11. Glass-Marmor L and Beitner R: Detachment of glycolytic enzymes from cytoskeleton of melanoma cells induced by calmodulin antagonists. Eur J Pharmacol 328: 241-248, 1997.

12. Schwartz D and Beitner R: Detachment of the glycolytic enzymes, phosphofructokinase and aldolase, from cytoskeleton of melanoma cells, induced by local anesthetics. Mol Genet Metab 69: 159-164, 2000.

13. Bozzetti F, Gavazzi C, Mariani L and Crippa F: Glucose-based total parenteral nutrition does not stimulate glucose uptake by human tumours. Clin Nutr 23: 417-421, 2004.

14. Tatsumi M, Nakamoto Y, Traughber B, Marshall LT, Geschwind JF and Wahl RL: Initial experience in small animal tumor imaging with a clinical positron emission tomography/ computed tomography scanner using 2-[F-18] fluoro-2-deoxyD-glucose. Cancer Res 63: 6252-6257, 2003.

15. Fukuda K, Taniguchi H, Koh T, Kunishima S and Yamagishi H: Relationships between oxygen and glucose metabolism in human liver tumours: positron emission tomography using (15)O and (18)F-deoxyglucose. Nucl Med Commun 25: 577-583, 2004.

16. Chen CJ, You SL, Lin LH, Hsu WL and Yang YW: Cancer epidemiology and control in Taiwan: A brief review. Jpn J Clin Oncol 32: S66-S81, 2002.

17. Schafer D, Hamm-Kunzelmann B and Brand K: Glucose regulates the promoter activity of aldolase A and pyruvate kinase M2 via dephosphorylation of Sp1. FEBS Lett 417: 325-328, 1997.

18. Kato K, Ogura T, Kishimoto A, Minegishi Y, Nakajima N, Miyazaki M and Esumi H: Critical roles of AMP-activated protein kinase in constitutive tolerance of cancer cells to nutrient deprivation and tumor formation. Oncogene 21: 6082-6090, 2002.

19. Izuishi K, Kato K, Ogura T, Kinoshita T and Esumi H: Remarkable tolerance of tumor cells to nutrient deprivation: possible new biochemical target for cancer therapy. Cancer Res 60: 6201-6207, 2000.

20. Mathupala SP, Rempel A and Pedersen PL: Glucose catabolism in cancer cells: identification and characterization of a marked activation response of the type II hexokinase gene to hypoxic conditions. J Biol Chem 276: 43407-43412, 2001.

21. Mathupala SP, Rempel A and Pedersen PL: Glucose catabolism in cancer cells. Isolation, sequence, and activity of the promoter for type II hexokinase. J Biol Chem 270: 16918-16925, 1995.

22. Goel A, Mathupala SP and Pedersen PL: Glucose metabolism in cancer. Evidence that demethylation events play a role in activating type II hexokinase gene expression. J Biol Chem 278: 15333-15340, 2003.

23. Rempel A, Bannasch P and Mayer D: Differences in expression and intracellular distribution of hexokinase isoenzymes in rat liver cells of different transformation stages. Biochim Biophys Acta 1219: 660-668, 1994.

24 Shinohara Y, Ichihara J and Terada H: Remarkably enhanced expression of the type II hexokinase in rat hepatoma cell line AH130. FEBS Lett 291: 55-57, 1991.

25. Rose IA and Warms JV: Stability of hexokinase II in vitro and in ascites tumor cells. Arch Biochem Biophys 213: 625-634, 1982.

26. Yasuda S, Arii S, Mori A, et al: Hexokinase II and VEGF expression in liver tumors: correlation with hypoxia-inducible factor 1 alpha and its significance. J Hepatol 40: 117-123, 2004.

27. Rempel A, Mathupala SP, Griffin CA, Hawkins AL and Pedersen PL: Glucose catabolism in cancer cells: amplification of the gene encoding type II hexokinase. Cancer Res 56: 2468-2471, 1996.

28. Mathupala SP, Heese C and Pedersen PL: Glucose catabolism in cancer cells. The type II hexokinase promoter contains functionally active response elements for the tumor suppressor p53. J Biol Chem 272: 22776-22780, 1997.

29. Izzo P, Costanzo P, Lupo A and Salvatore F: Novel Aspects of the Human Aldolase Gene Family and its Expression in Tumor Cells. In: Human tumor markers. Cimino F, Birkmayer GD, Klavins JV, Pimentel E and Salvatore F (eds). Walter de Gruyter, Berlin, pp889-898, 1987. 
30. Asaka M, Kimura T, Meguro T, Kato M, Kudo M, Miyazaki T and Alpert E: Alteration of aldolase isozymes in serum and tissues of patients with cancer and other diseases. J Clin Lab Anal 8: 144-148, 1994

31. Motomura M, Mukai T, Ozaki I, Joh K, Arai Y, Sakai T and Hori K: Transcriptional regulation of an aldolase gene in the regenerating rat liver. Jpn Gastroenterol 25: 350-355, 1990.

32. Bustamante E, Morris HP and Pedersen PL: Energy metabolism of tumor cells. Requirement for a form of hexokinase with a propensity for mitochondrial binding. J Biol Chem 256: 8699-8704, 1981.

33. Kinoshita $M$ and Miyata M: Underexpression of mRNA in human hepatocellular carcinoma focusing on eight loci. Hepatology 36: 433-438, 2002.

34. Hsu HC, Chiou TJ, Chen JY, Lee CS, Lee PH and Peng SY: Clonality and clonal evolution of hepatocellular carcinoma with multiple nodules. Hepatology 13: 923-928, 1991.

35. Pan HW, Ou YH, Peng SY, et al: Overexpression of osteopontin is associated with intrahepatic metastasis, early recurrence, and poorer prognosis of surgically resected hepatocellular carcinoma. Cancer 98: 119-127, 2003.

36. Peng SY, Chen WJ, Lai PL, Jeng YM, Sheu JC and Hsu HC: High alpha-fetoprotein level correlates with high stage, early recurrence and poor prognosis of hepatocellular carcinoma: significance of hepatitis virus infection, age, p53 and betacatenin mutations. Int J Cancer 112: 44-50, 2004.

37. Pan HW, Chou HY, Liu SH, Peng SY, Liu CL and Hsu HC: Role of L2DTL, cell cycle-regulated nuclear and centrosome protein, in aggressive hepatocellular carcinoma. Cell Cycle 5: 2676-2687, 2006

38. Yuan RH, Jeng YM, Chen HL, et al: Stathmin overexpression cooperates with p53 mutation and osteopontin overexpression, and is associated with tumour progression, early recurrence, and poor prognosis in hepatocellular carcinoma. J Pathol 209: $549-558,2006$

39. Hsu HC, Tseng HJ, Lai PL, Lee PH and Peng SY: Expression of p53 gene in 184 unifocal hepatocellular carcinomas: association with tumor growth and invasiveness. Cancer Res 53: 4691-4694, 1993.
40. Hsu HC, Peng SY, Lai PL, Chu JS and Lee PH: Mutations of p53 gene in hepatocellular carcinoma (HCC) correlate with tumor progression and patient prognosis. Int J Oncol 4: 1341-1347, 1994

41. Hu RH, Lee PH, Yu SC, et al: Surgical resection for recurrent hepatocellular carcinoma: prognosis and analysis of risk factors. Surgery 120: 23-29, 1996.

42. Poon RT, Fan ST, Ng IO, Lo CM, Liu CL and Wong J: Different risk factors and prognosis for early and late intrahepatic recurrence after resection of hepatocellular carcinoma. Cancer 89: 500-507, 2000.

43. Gwak GY, Yoon JH, Kim KM, Lee HS, Chung JW and Gores GJ: Hypoxia stimulates proliferation of human hepatoma cells through the induction of hexokinase II expression. J Hepatol 42: 358-364, 2005

44. Hollstein M, Sidransky D, Vogelstein B and Harris CC: p53 mutations in human cancers. Science 253: 49-53, 1991.

45. Levine AJ, Momand J and Finlay CA: The p53 tumour suppressor gene. Nature 351: 453-456, 1991.

46. Vogelstein B, Kinzler KW: p53 function and dysfunction. Cell 70: 523-526, 1992.

47. Zhang W, Shay JW and Deisseroth A: Inactive p53 mutants may enhance the transcriptional activity of wild-type p53. Cancer Res 53: 4772-4775, 1993.

48. Park DJ, Nakamura H, Chumakov AM, Said JW, Miller CW, Chen DL and Koeffler HP: Transactivational and DNA binding abilities of endogenous p53 in p53 mutant cell lines. Oncogene 9: 1899-1906, 1994.

49. Koonin EV, Rogozin IB and Glazko GV: p53 gain-of-function: tumor biology and bioinformatics come together. Cell Cycle 4 686-688, 2005

50. Di Agostino S, Strano S, Emiliozzi V, et al: Gain of function of mutant p53: the mutant p53/NF-Y protein complex reveals an aberrant transcriptional mechanism of cell cycle regulation. Cancer Cell 10: 191-202, 2006. 